\title{
Biotyping of Enterobacter cloacae
}

\author{
DAVID C OLD
}

\begin{abstract}
From the Department of Bacteriology, University of Dundee Medical School, Ninewells Hospital, Dundee DDI $9 S Y$
\end{abstract}

SUMMARY A biotyping scheme for Enterobacter cloacae is proposed. Tests with seven substrates that gave reliable, reproducible results with 110 isolates of $E$ cloacae formed the basis of the biotyping scheme which would allow recognition of 128 potential biotypes.

Among members of the tribe Klebsielleae, klebsiellae and serratiae are generally considered to be more commonly implicated in hospital-acquired infections than are enterobacters. ' Accordingly, for the former groups, well-established methods for type discrimination within the species are available. ${ }^{2-5}$ The occurrence of an outbreak due to Enterobacter cloacae revealed the lack of a suitable system for type discrimination within that species. Our previous experience with other enterobacteria ${ }^{67}$ suggested that biotyping might provide an easy, reproducible system for that purpose; the basis of such a scheme is reported in this paper.

\section{Material and methods}

ORGANISMS: ISOLATION AND IDENTIFICATION Most $(85 \%)$ of the 105 clinical isolates of $E$ cloacae examined were recovered from specimens originating from one maternity ward in a Dundee hospital during the three months from September to November 1978; the remainder (16 cultures) were stock cultures of $E$ cloacae from that or other wards isolated before or after the "epidemic" period. Most of the cultures $(42 \%)$ were isolated from vaginal or placental swabs; the remainder were from diverse clinical specimens including blood, expressed breast milk, faeces, urine, and pus from wounds. Five reference strains of $E$ cloacae from the National Collection of Type Cultures, Colindale, London, were included: NCTC strains 9529, 9711, 9785, 9842 and 10005.

Presumptive coliform organisms present on diagnostic platings of clinical specimens on MacConkey Agar (Oxoid Ltd) were picked and identified as $E$ cloacae by standard techniques. ${ }^{8}$ The reactions of isolates judged to be $E$ cloacae were uniform in the following tests: motile, indole-negative, ureasenegative, Simmons's citrate-positive, arginine

Accepted for publication 5 January 1982 dihydrolase-positive, lysine decarboxylase-negative and ornithine decarboxylase-positive.

\section{BIOTYPING MEDIA}

The ability of isolates to ferment sugars or sugar alcohols was tested in peptone water (Oxoid CM9) medium, pH $6 \cdot 8$, with $0.002 \%$ (wt/vol) bromocresol purple as indicator, dispensed in $5 \mathrm{ml}$ amounts in screw-capped, quarter-ounce $(7 \mathrm{~g})$ bottles. The substrates tested, at a final concentration of $1 \%$ (wt/vol) were: adonitol, dulcitol, inositol, $\alpha$-methylglucoside, rhamnose and salicin. The preparation of aesculin agar $^{7}$ and malonate broth ${ }^{8}$ have been described elsewhere.

\section{INOCULATION OF MEDIA}

A well-isolated colony from a pure culture of the test organism was spread on nutrient agar and after incubation for $24 \mathrm{~h}$ at $37^{\circ} \mathrm{C}$, suspended (to $10^{\circ}$ bacteria/ml) in saline $(\mathrm{NaCl}, 7.5 \mathrm{~g} / \mathrm{l})$. Biotyping media were inoculated with $0.02 \mathrm{ml}$ of that suspension.

\section{INTERPRETATION OF TESTS}

All cultures were incubated at $37^{\circ} \mathrm{C}$. Fermentation tests were examined daily for up to seven days for the production of acid (indicator change to yellow) in cultures of the test organism. As a result of these observations it became clear that tests for the fermentation of each carbohydrate were best evaluated at $24 \mathrm{~h}$, the definitive time giving optimal separation of strains into genotypically fermenting and nonfermenting types. Utilisation of malonate was indicated by a colour change of indicator to deep blue after incubation for 24 and $48 \mathrm{~h}$. Hydrolysis of aesculin in plate tests was indicated by the presence of black precipitate around areas of growth. In each typing test, results were recorded as positive $(+)$ or negative (-) at the definitive time of reading. The biotype was expressed as a profile of positive and negative results (Table). 
Reactions of 110 isolates of Enterobacter cloacae in seven biotyping tests

\begin{tabular}{|c|c|c|c|c|c|c|c|c|c|c|c|c|c|}
\hline \multirow[b]{2}{*}{ Test } & \multicolumn{13}{|c|}{ Biotype profile } \\
\hline & $l$ & 2 & 3 & 4 & 5 & 6 & 7 & 8 & 9 & 10 & 11 & 12 & 13 \\
\hline \multicolumn{14}{|l|}{ Fermentation of: } \\
\hline Adonitol & + & + & - & - & - & - & - & - & - & - & - & - & - \\
\hline Dulcitol & - & - & + & + & + & + & - & - & - & - & - & - & - \\
\hline Inositol & + & - & + & - & - & - & - & - & - & - & - & - & - \\
\hline$\alpha$-methylglucoside & + & + & + & + & - & - & + & + & - & - & - & - & - \\
\hline Rhamnose & + & + & + & - & + & + & + & + & + & + & + & + & + \\
\hline \multicolumn{14}{|l|}{ Utilisation of: } \\
\hline Malonate & + & + & + & - & + & - & + & - & + & + & - & - & + \\
\hline Aesculin* & + & - & + & + & + & - & - & + & + & - & + & - & + \\
\hline No of isolates & 1 & 6 & 1 & 86 & 1 & 1 & 2 & 1 & 2 & 5 & 1 & 2 & 1 \\
\hline
\end{tabular}

*Or salicin.

\section{Results}

\section{DISCRIMINATION}

Biotyping of 110 isolates of $E$ cloacae by their reactions in tests with eight substrates (Table) distinguished 13 biotypes, the most common being that represented by 86 replicate isolates of the "epidemic" strain (type 4, Table).

Differentiation of the isolates into fermenting and non-fermenting types in tests with the three sugar alcohols and the methyl sugars, $\alpha$-methylglucoside and rhamnose, was best achieved with a definitive time of reading of $24 \mathrm{~h}$. In order that the interpretation of the results should not be biased by consideration of such a large number of isolates (86) of the same strain, only one isolate of the epidemic strain will be considered in assessing the degree of discrimination achieved by the different tests. Thus, with reference to 25 strains (rather than 110 isolates) it was clear that different degrees of differentiation were obtained as follows: with $\alpha$-methylglucoside, discrimination was excellent ( $48 \%$ of isolates fermenting (positive); $52 \%$ of isolates non-fermenting (negative)); with adonitol ( $28 \%$ positive; $72 \%$ negative) and dulcitol (16\% positive; $84 \%$ negative) it was moderate; and, with inositol and rhamnose, each of which discriminated only $8 \%$ of the minority type (respectively, positive and negative), it was poor. On occasion, positive results were obtained between two and seven days of incubation in some tests with cultures that were negative at $24 \mathrm{~h}$, a finding that indicated the origin of mutant fermenting types in the course of extended incubation in selective medium. Accordingly, with reference to the definitive time of reading, these irregular "latepositive" results were scored as negative.

Strong utilisers of malonate invariably gave positive results on testing of different colonies from the same culture at the same time and testing of cultures of the same isolate at different times. Some cultures which at $24 \mathrm{~h}$ were malonate-negative gave positive results irregularly when readings were at $48 \mathrm{~h}$. Thus, although the latter time is often recommended for tests with malonate, best differentiation with this series of E cloacae was achieved at $24 \mathrm{~h}$, at which time discrimination was moderately good $(76 \%$ positive; $24 \%$ negative).

A marked correlation between ability of $E$ cloacae strains to ferment salicin in peptone water and to hydrolyse aesculin in agar was noted, when tests were read at $24 \mathrm{~h}$. The single isolate which gave discrepant results fermented salicin, but did not hydrolyse aesculin, in $24 \mathrm{~h}$.

\section{REPRODUCIBILITY}

When each of 110 isolates was tested on three different occasions over three years, discrepancies in results were not observed on any occasion for any culture for fermentation tests or malonate tests read at their definitive times. The least satisfactory test in terms of reproducibility was that for salicin hydrolysis in peptone water; thus, some nonhydrolysing isolates gave positive results on some occasions of testing. This test gave reproducible results, however, when several (up to six) colonies from each culture were tested and the results given by the majority of colonies taken. The irregular positive results obtained with some cultures of salicin non-hydrolysing isolates were probably due to the presence of hydrolysing mutants in the cultures. Similar anomalies have been noted with salicin testing of Escherichia coli. ' On the other hand, discrepancies were not observed with the aesculin test which was chosen, therefore, as the test more likely to give reproducible results. On the basis of the results with aesculin hydrolysis, discrimination was good ( $36 \%$ positive; $64 \%$ negative).

\section{Discussion}

Because members of the Klebsielleae are often antibiotic resistant, their association with infections in 
hospitalised patients presents considerable problems. The recognition of the importance of this particular group of organisms in nosocomial infections has stimulated the development of different kinds of typing methods for those members more usually implicated in hospital infections. ${ }^{2-5}$ The species of Enterobacter: aerogenes, cloacae and sakazakii ${ }^{10}$ have, however, received less attention. ${ }^{1}$

The commonly recommended method for typing E cloacae, for example, is serotyping which allows recognition of more than 70 serotypes. ${ }^{11}$ That method, however, is unlikely to prove acceptable in most laboratories because commercial sera are not available and the establishment of the system de novo and the performance of the tests, is likely to prove burdensome and time-consuming. Biotyping of $E$ cloacae, if feasible, would provide with simple reagents an inexpensive method for rapid typing, as it did for $E$ aerogenes ${ }^{1}$ and $E$ sakazakii. ${ }^{10}$

In the establishment of a biotyping scheme for any bacterial species, tests suitable for identification of the species must be distinguished from those tests suitable for the discrimination of types within a species. Thus, discriminatory tests separate isolates into phenotypically positive or negative types. Though optimal separation $(50 \%$ positive; $50 \%$ negative) is rarely achieved, tests may still be used for biotyping even when the separation they achieve departs considerably from the optimum. In the present study, each test afforded strain discrimination; some-for example, inositol and rhamnose, were less satisfactory than others-for example, $\alpha$-methylglucoside. The strong correlation found between the ability to hyrolyse aesculin and salicin was not unexpected since the same $\beta$-glucosidase is known to hydrolyse these two structurally related compounds. ${ }^{12}$ In view of the observed discrepancies with the salicin test, the aesculin test is to be preferred. At the definitive time of reading, only two phenotypes $(+,-)$ were considered for each test and so the scheme, as presented, allowed recognition of 128 potential biotypes with the use of the seven substrates: adonitol, dulcitol, inositol, $\alpha$-methylglucoside, rhamnose, malonate, and aesculin. This series of isolates, though primarily of local origin, was represented, nevertheless, by 13 biotypes; and each of the five NCTC reference strains $(9529,9711,9785,9842$ and 10005) belonged to different biotypes $(3,5,2,13$ and 7 , respectively).

In typing for the discrimination of members of a bacterial species, it is important that: (i) most of the isolates are typeable, (ii) there is a high discriminating ability, (iii) reliability and reproducibility are achieved, and (iv) tests are simple to perform. Each point was fulfilled with the present system, and the phenotypic diversity demonstrated among this series of isolates indicates the potential value of biotyping for $E$ cloacae.

It was not the purpose of the present paper on E cloacae to discuss the clinical or epidemiological aspects of the outbreak, the details of which will be presented elsewhere (D Parratt, R Traynor, personal communication 1981). Nevertheless, it should be noted that the epidemic strain (represented by 86 isolates) was unusual in being dulcitol-fermenting and rhamnose non-fermenting and, hence, was readily discriminated from other types of Ecloacae which are generally dulcitol non-fermenting $(87 \%)$ and rhamnose-fermenting $(92 \%){ }^{8}$ Though of a rare biotype, the strain readily identified as $E$ cloacae by its reactions in tests with arginine, lysine and ornithine $(+,-,+$, respectively). Furthermore, by its lack of a yellow pigment, its fermentation of sorbitol and its resistance to ampicillin and cephaloridine (DC Old, unpublished results), it was clear that it was not an isolate of $E$ sakazakii which has many biochemical properties in common with E cloacae, ${ }^{10}$ and may, on occasion, be associated with conditions of clinical significance. ${ }^{1013}$

Thus, biotyping would seem to have potential value for discriminating types of E cloacae. It allowed identification of the epidemic strain in this outbreak; its application in future epidemiological studies remains to be evaluated.

I wish to thank Mr R Traynor for the gift of isolates of $E$ cloacae and information about their clinical origin; without that assistance, this study would not have been possible. Discussion with Professor JP Duguid is gratefully acknowledged. Mrs J Robertson gave valuable technical assistance.

\section{References}

' Barr JG, Hogg GM. Biotypes of Klebsiella pneumoniae (sensu lato) and Enterobacter aerogenes characterised by differential substrate metabolism: application of the technique. J Clin Pathol 1979;32:935-43.

${ }^{2}$ Grimont PAD, Grimont F. Biotyping of Serratia marcescens and its use in epidemiological studies. J Clin Microbiol 1978:8:73-83.

${ }^{3}$ Haverkorn ML, Michel MF. Nosocomial klebsiellas I. Colonization of hospitalized patients. J Hyg 1979;82:177-93.

4 Yu VL. Serratia marcescens. Historical perspective and clinical review. N Engl J Med 1979;300:887-93.

${ }^{5}$ Pitt TL, Erdman YJ, Bucher C. The epidemiological type identification of Serratia marcescens from outbreaks of infection in hospitals. J Hyg 1980;84:269-83.

- Duguid JP, Anderson ES, Alfredsson GA, Barker R, Old DC. A new biotyping scheme for Salmonella typhimurium and its phylogenetic significance. J Med Microbiol 1975;8:149-66.

${ }^{7}$ Crichton PB, Old DC. Biotyping of Escherichia coli. J Med Microbiol 1979;12:473-86.

sdwards PR, Ewing WH. Identification of Enterobacteriaceae. 3rd ed. Minneapolis, Minnesota: Burgess, 1972. 
'Sakazaki R. Genus VII Enterobacter. In: Buchanan RE, Gibbons NE, eds. Bergey's manual of determinative bacteriology. 8th ed. Baltimore: Williams and Wilkins, 1974:324-5.

${ }^{10}$ Farmer JJ III, Asbury MA, Hickman FW, Brenner DJ. Enterobacteriaceae study group. Enterobacter sakazakii: a new species of "Enterobacteriaceae" isolated from clinical specimens. Int J Syst Bacteriol 1980;30:569-84.

"Sakazaki R, Namioka S. Serological studies on the Cloaca (Aerobacter) group of enteric bacteria. Jpn J Med Sci Biol 1960;13:1-12.
${ }^{12}$ Edberg SC, Pittman S, Singer JM. Esculin hydrolysis by Enterobacteriaceae. J Clin Microbiol 1977;6:111-6.

${ }^{13}$ Kleiman MB, Allen SD, Neal P, Reynolds J. Meningoencephalitis and compartmentalization of the cerebral ventricles caused by Enterobacter sakazakii. J Clin Microbiol $1981 ; 14: 352-4$.

Requests for reprints to: Dr DC Old, Department of Bacteriology, University of Dundee Medical School, Ninewells Hospital, Dundee DD1 9SY, Scotland. 